\title{
Kelvin probe force microscopy by direct dissipative electrostatic force modulation
}

\author{
Yoichi Miyahara. ${ }^{*}$ Jessica Topple, Zeno Schumacher, and Peter Grutter \\ Department of Physics, Faculty of Science, McGill University, Montreal, Quebec, Canada H3A 2T8
}

(Dated: July 15, 2021)

\begin{abstract}
We report a new experimental technique for Kelvin probe force microscopy (KPFM) using the dissipation signal of frequency modulation atomic force microscopy for bias voltage feedback. It features a simple implementation and faster scanning as it requires no low frequency modulation. The dissipation is caused by the oscillating electrostatic force that is coherent with the tip oscillation, which is induced by a sinusoidally oscillating voltage applied between the tip and sample. We analyzed the effect of the phase of the oscillating force on the frequency shift and dissipation and found that the relative phase of $90^{\circ}$ that causes only the dissipation is the most appropriate for KPFM measurements. The present technique requires a significantly smaller ac voltage amplitude by virtue of enhanced force detection due to the resonance enhancement and the use of fundamental flexural mode oscillation for electrostatic force detection. This feature will be of great importance in the electrical characterizations of technically relevant materials whose electrical properties are influenced by the externally applied electric field as is the case in semiconductor electronic devices.
\end{abstract}

\section{INTRODUCTION}

Kelvin probe force microscopy (KPFM), a variant of atomic force microscopy (AFM) has become one of the indispensable tools used to investigate electronic properties of nanoscale material as well as nanoscale devices. In KPFM, a surface potential of a sample is measured by detecting a capacitive electrostatic force that is a function of the surface potential and applied bias voltage. In order to separate the electrostatic force component from other force components such as van der Waals force, chemical bonding force and magnetic force, the electrostatic force is modulated by applying an ac bias voltage and the resulting modulated component of the measured force is detected by lock-in detection [1].

KPFM has been implemented in a variety of ways that can be classified into two distinct categories, amplitude modulation (AM) [2-4] and frequency modulation (FM) [4, 5]. The former implementation takes advantage of enhanced electrostatic force detection sensitivity by tuning the modulation frequency to one of the resonance frequencies of the AFM cantilever, leading to an enhanced detection of the electrostatic force by its quality $(Q-)$ factor that can reach over 10,000 in vacuum [6].

The latter method (FM-KPFM) detects the modulation in the resonance frequency shift that is induced by a low frequency ac voltage. While this method requires a much higher ac voltage amplitude, it offers higher spatial resolution because the resonance frequency shift is determined by the electrostatic force gradient with respect to the tip-sample distance rather than the force itself [3, 4, 7].

Here we report a new KPFM implementation (D-KPFM) using the dissipation signal of conventional FM-AFM systems to detect the electrostatic force. The dissipation arises from an oscillating electrostatic force acting on the AFM tip that is coherent with the tip oscillation (trajectory). The oscillating electrostatic force is induced by applying an ac voltage between the tip and sample. By setting the phase of the ac voltage to $90^{\circ}$ out of phase with respect to the tip oscillation, an electrically induced dissipation can result without affecting the resonance frequency shift, allowing topography imaging to be performed in constant frequency shift mode. The KPFM feedback loop can be implemented with the electrically induced dissipation signal as it is proportional to the effective dc potential difference between the tip and sample, $\left(V_{\text {bias }}-V_{\text {cpd }}\right)$, where $V_{\text {bias }}$ and $V_{\text {cpd }}$ are an applied dc voltage and the contact potential difference, respectively.

This new technique makes it possible to take full advantage of the enhanced force sensitivity by the high $Q$-factor fundamental resonance mode as there is no need to excite higher flexural modes for the electrostatic force detection. In conventional AM-KPFM [2, 3], because the fundamental mode is used for topography imaging, the frequency of ac bias voltage is typically configured to excite a higher resonance mode (typically second flexural mode) that have much higher effective spring constant, cancelling the high $Q$-factor resonance enhancement. The enhanced sensitivity of the technique presented here allows the use of a much smaller ac voltage enabling less invasive potential measurements, which is of great importance in the electrical characterizations of technically relevant materials whose electrical properties are influenced by the externally applied electric field as is the case in semiconductor electronic devices.

Although a similar technique has already been reported by Fukuma et al. [8], it has not been adopted widely probably because of complexity in its implementation. The present technique is simpler in implementation and even requires no additional lock-in amplifier as there is no low-frequency modulation involved.

\section{THEORY}

The electrostatic force between two conductors connected to an ac and dc voltage source, $F_{\text {elec}}$, is described as follows 
[9]:

$$
\begin{aligned}
F_{\text {elec }}= & -\frac{1}{2} \frac{\partial C}{\partial z}\left\{V_{\text {bias }}-V_{\mathrm{cpd}}+V_{\mathrm{ac}} \cos \left(\omega_{\mathrm{el}} t+\phi\right)\right\}^{2} \\
= & \alpha\left\{V_{\mathrm{dc}}+V_{\mathrm{ac}} \cos \left(\omega_{\mathrm{el}} t+\phi\right)\right\}^{2} \\
= & F_{0}+F_{1}+F_{2} \\
& \alpha \equiv-\frac{1}{2} \frac{\partial C}{\partial z}, V_{\mathrm{dc}} \equiv V_{\text {bias }}-V_{\mathrm{cpd}}
\end{aligned}
$$

where $C$ is the tip-sample capacitance, $V_{\text {bias }}$ and $V_{\text {cpd }}$ are the applied dc voltage and the contact potential difference, and $V_{\mathrm{ac}}, \omega_{\mathrm{el}}$ and $\phi$ are the amplitude, angular frequency and phase of the ac bias voltage. $z$ is the position of the tip with respect to the sample surface and the oscillating tip around its mean position, $z_{0}$, expressed as $z(t)=z_{0}+A \cos \left(\omega_{\mathrm{m}} t\right)$ with $\omega_{\mathrm{m}}$ and $A$ being its oscillation angular frequency and amplitude, respectively. We assume that the tip oscillation is driven by another means such as piezoacoustic or photothermal excitation and its frequency, $f_{\mathrm{m}}=\omega_{\mathrm{m}} / 2 \pi$, is chosen to be that of the fundamental flexural resonance mode, $f_{0}$.

Expanding Eq. 1 and isolating each harmonic component, $F_{0}, F_{1}$ and $F_{2}$, which result from the applied ac bias voltage, yields the following terms:

$$
\begin{aligned}
& F_{0}=\alpha\left(V_{\mathrm{dc}}^{2}+\frac{V_{\mathrm{ac}}^{2}}{2}\right) \\
& F_{1}=2 \alpha V_{\mathrm{dc}} V_{\mathrm{ac}} \cos \left(\omega_{\mathrm{el}} t+\phi\right) \\
& F_{2}=\frac{1}{2} \alpha V_{\mathrm{ac}}^{2} \cos \left\{2\left(\omega_{\mathrm{el}} t+\phi\right)\right\}
\end{aligned}
$$

Notice that the $z$-dependence of $\alpha=-\frac{1}{2} \frac{\partial C}{\partial z}(z)$ must be taken into account in order to correctly describe the response of the oscillating cantilever subject to the oscillating electrostatic force induced by the coherent ac voltage.

By expanding $\alpha$ around the mean position, $z_{0}$, and taking the first order term, $\alpha$ is expressed as follows:

$$
\alpha(z) \approx \alpha\left(z_{0}\right)+\alpha^{\prime}\left(z-z_{0}\right)=\alpha_{0}+\alpha^{\prime} A \cos \left(\omega_{\mathrm{m}} t\right)
$$

Substituting eq. 5 into eq. 2 and rearranging it yields,

$$
\begin{aligned}
F_{0}(t) & =\left\{\alpha_{0}+\alpha^{\prime} A \cos \left(\omega_{\mathrm{m}} t\right)\right\}\left(V_{\mathrm{dc}}^{2}+\frac{V_{\mathrm{ac}}^{2}}{2}\right) \\
& =\alpha_{0}\left(V_{\mathrm{dc}}^{2}+\frac{V_{\mathrm{ac}}^{2}}{2}\right)+\alpha^{\prime} A\left(V_{\mathrm{dc}}^{2}+\frac{V_{\mathrm{ac}}^{2}}{2}\right) \cos \left(\omega_{\mathrm{m}} t\right)
\end{aligned}
$$

Note that the second term expresses an oscillating force whose frequency is $\omega_{\mathrm{m}} / 2 \pi$ while the first term expresses the static deflection of the cantilever. As this oscillating force is in phase with respect to $z(t)$, it results in a shift in resonance frequency [10].

Substituting eq. 5 into the expression of $F_{1}$ (Eq. 3 ) gives

$$
\begin{aligned}
& F_{1}(t)=2\left\{\alpha_{0}+\alpha^{\prime} A \cos \left(\omega_{\mathrm{m}} t\right)\right\} V_{\mathrm{dc}} V_{\mathrm{ac}} \cos \left(\omega_{\mathrm{el}} t+\phi\right) \\
& \quad=2 \alpha_{0} V_{\mathrm{dc}} V_{\mathrm{ac}} \cos \left(\omega_{\mathrm{el}} t+\phi\right) \\
& \left.+\alpha^{\prime} A V_{\mathrm{dc}} V_{\mathrm{ac}}\left[\cos \left\{\left(\omega_{\mathrm{el}}+\omega_{\mathrm{m}}\right) t+\phi\right)\right\}+\cos \left\{\left(\omega_{\mathrm{el}}-\omega_{\mathrm{m}}\right) t-\phi\right\}\right]
\end{aligned}
$$

When $\omega_{\mathrm{el}} \ll \omega_{\mathrm{m}}$, a pair of sideband peaks appear around the mechanical resonance peak due to the second and third terms of eq. 7. which are detected in the conventional FM-KPFM [4].

We focus on a special case where $\omega_{\mathrm{el}}=\omega_{\mathrm{m}} . F_{1}(t)$ can be simplified as follows:

$$
\begin{aligned}
& F_{1}(t)=\alpha^{\prime} A V_{\mathrm{dc}} V_{\mathrm{ac}} \cos \phi+2 \alpha_{0} V_{\mathrm{dc}} V_{\mathrm{ac}} \cos \left(\omega_{\mathrm{m}} t+\phi\right) \\
& +\alpha^{\prime} A V_{\mathrm{dc}} V_{\mathrm{ac}} \cos \left(2 \omega_{\mathrm{m}} t+\phi\right)
\end{aligned}
$$

We notice that the interaction between the mechanical oscillation of the tip and the oscillating electrostatic force produce a static force and two harmonic forces with frequency, $\omega_{\mathrm{m}}$ and $2 \omega_{\mathrm{m}}$.

Likewise $F_{2}(t)$ (eq. 4 ) contains three harmonic terms with their frequency, $\omega_{\mathrm{m}}, 2 \omega_{\mathrm{m}}$ and $3 \omega_{\mathrm{m}}$ under the same condition $\left(\omega_{\mathrm{m}}=\omega_{\mathrm{el}}\right)$ as follows:

$$
\begin{aligned}
F_{2}(t) & =\frac{1}{2}\left\{\alpha_{0}+\alpha^{\prime} A \cos \left(\omega_{\mathrm{m}} t\right)\right\} V_{\mathrm{ac}}^{2} \cos \left\{2\left(\omega_{\mathrm{el}} t+\phi\right)\right\} \\
& =\frac{1}{2} \alpha_{0} V_{\mathrm{ac}}^{2} \cos \left\{2\left(\omega_{\mathrm{m}} t+\phi\right)\right\} \\
& +\frac{1}{4} \alpha^{\prime} A V_{\mathrm{ac}}^{2}\left\{\cos \left(\omega_{\mathrm{m}} t+2 \phi\right)+\cos \left(3 \omega_{\mathrm{m}} t+2 \phi\right)\right\}
\end{aligned}
$$

As is shown in the theory of FM-AFM [10-12], the resonance frequency shift and dissipation signal are essentially determined by the in-phase and quadrature component of the fundamental harmonic component (in this case $\omega_{\mathrm{m}}$ ) of the oscillating force, respectively. Putting together all the $\omega_{\mathrm{m}}$ components from $F_{0}, F_{1}$ and $F_{2}$ and rearranging them, we get the following expression:

$$
F_{\omega}(t)=F_{\text {in }} \cos \omega_{\mathrm{m}} t+F_{\text {quad }} \sin \omega_{\mathrm{m}}
$$

where

$$
\begin{aligned}
F_{\text {in }}= & \alpha^{\prime} A\left(V_{\mathrm{dc}}+\frac{\alpha_{0} \cos \phi}{\alpha^{\prime} A} V_{\mathrm{ac}}\right)^{2} \\
- & {\left[\frac{\alpha_{0}^{2} \cos ^{2} \phi}{\alpha^{\prime} A}-\frac{\alpha^{\prime} A}{2}\left(1+\frac{\cos 2 \phi}{2}\right)\right] V_{\mathrm{ac}}^{2} } \\
F_{\text {quad }}=- & 2 \alpha_{0} V_{\mathrm{dc}} V_{\mathrm{ac}} \sin (\phi)-\frac{1}{2} \alpha^{\prime} A V_{\mathrm{ac}}^{2} \sin (\phi) \cos (\phi)
\end{aligned}
$$

These $F_{\text {in }}$ and $F_{\text {quad }}$ cause the resonance frequency shift and dissipation signal in FM-AFM, respectively. As can be seen in the formula for $F_{\text {in }}$, the frequency shift versus $V_{\mathrm{dc}}$ curve will be a parabola whose minimum is shifted from $V_{\text {cpd }}$ by the value determined by the phase, $\phi$, and amplitude, $V_{\mathrm{ac}}$, of the oscillating bias voltage. This indicates that the bias voltage at the parabola minimum is no longer $V_{\text {cpd }}$. However, in the special case where $\phi=90^{\circ}$, we find

$$
\begin{aligned}
F_{\text {in }} & =\alpha^{\prime} A\left(V_{\mathrm{dc}}^{2}+\frac{V_{\mathrm{ac}}^{2}}{4}\right) \\
F_{\text {quad }} & =-2 \alpha_{0} V_{\mathrm{dc}} V_{\mathrm{ac}}=-2 \alpha_{0}\left(V_{\text {bias }}-V_{\mathrm{cpd}}\right) V_{\mathrm{ac}}
\end{aligned}
$$




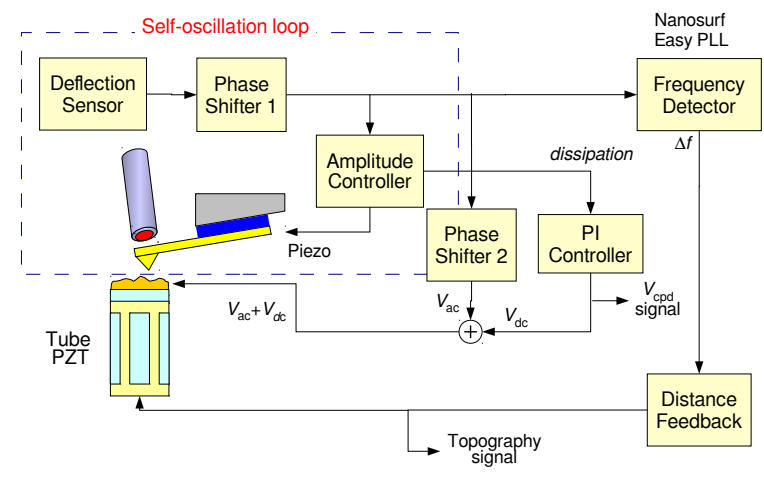

Figure 1. Block diagram of the experimental setup for D-KPFM measurements.

The resulting resonance frequency shift, $\Delta f$ and the dissipation signal, $g$, are obtained using the formulas found in Ref. $10-12$ as follows:

$$
\begin{aligned}
\Delta f & =-\frac{1}{2} \frac{f_{0}}{k} \frac{F_{\text {in }}}{A}=-\frac{1}{2} \frac{f_{0}}{k} \alpha^{\prime}\left\{\left(V_{\text {bias }}-V_{\mathrm{cpd}}\right)^{2}+\frac{V_{\mathrm{ac}}^{2}}{4}\right\} \\
g & =g_{0}\left(1-\frac{Q}{k A} F_{\text {quad }}\right)=g_{0}\left\{1+2 \frac{Q}{k A} \alpha_{0}\left(V_{\text {bias }}-V_{\text {cpd }}\right) V_{\mathrm{ac}}\right\}
\end{aligned}
$$

where $k$ is the effective spring constant of the fundamental flexural mode of the cantilever and $g_{0}$ is the dissipation without the ac bias voltage which is given by the mechanical $Q$ factor of the cantilever. In the case where the applied bias, $V_{\text {bias }}$, is equal to the contact potential difference, $V_{\text {cpd }}$, the dissipation goes back to its original value, $g_{0}$. It is therefore possible to use the dissipation signal, $g$, as the KPFM bias voltage feedback signal with $g_{0}$ as its control setpoint value.

\section{EXPERIMENTAL}

Figure 11 depicts the block diagram of the experimental setup used for D-KPFM measurements. As we notice, the DKPFM technique requires only two additional components, a phase shifter and proportional-integrator (PI) controller compared to normal FM-AFM systems. The oscillation of the AFM cantilever was controlled by a self-oscillation feedback loop electronics which consists of a phase shifter and an amplitude controller. The amplitude controller is used to maintain a constant oscillation amplitude and composed of a rootmean-square (RMS) amplitude detector and a PI controller (NanoSurf easyPLLplus oscillator controller). The output of the amplitude feedback PI controller is the dissipation signal which will be used for controlling the dc bias voltage. The detection bandwidth of the RMS amplitude detector was ex- tended to about $10 \mathrm{kHz}$ by replacing the integration capacitor in the original RMS detector circuit.

The deflection signal is fed into the additional phase shifter, which serves to adjust the relative phase, $\phi$, to produce the ac voltage which is $90^{\circ}$ out of phase to the cantilever deflection. Due to the phase delay in the deflection sensing electronics, the actual phase shift value set by the phase shifter may be different from $90^{\circ}$. The dissipation signal acts as the input signal to the PI controller, which adjusts the applied dc bias, $V_{\text {bias }}$, to maintain a constant dissipation equal to the value without $V_{\mathrm{ac}}$ applied, $g_{0}$.

We used a JEOL JSPM-5200 atomic force microscope for the experiments with the modifications described below. The original laser diode was replaced by a fiber-optic collimator with a focusing lens that is connected to a fiber-coupled laser diode module (OZ Optics). The laser diode was mounted on a temperature controlled fixture and its driving current was modulated with a radio frequency signal to reduce the deflection detection noise [13]. The bias voltage was applied to the sample with reference to the grounded AFM tip to reduce the effect of the capacitive crosstalk of $V_{\text {ac }}$ to the cantilever excitation piezo [14, 15]. The excitation piezo was shunted with a chip resistor with low resistance $(\sim 10 \Omega)$ to further reduce the effect. The original controller was replaced with a opensource controller, GXSM, [16] with the dedicated acquisition hardware (MK2-A810, SoftdB).

A commercial silicon AFM cantilever (NSC15, MikroMasch) with a typical spring constant of $20 \mathrm{~N} / \mathrm{m}$ and resonance frequency of $\sim 300 \mathrm{kHz}$ was used in high-vacuum environment with the pressure of $1 \times 10^{-7}$ mbar. The oscillation amplitude and quality factor of the cantilever used for the DKPFM imaging were $5 \mathrm{~nm}_{\mathrm{p}-\mathrm{p}}$ and $\sim 5500$, respectively.

\section{RESULTS AND DISCUSSION}

Figure 2 shows simultaneously measured $\Delta f$ and $g$ versus $V_{\text {bias }}$ curves with a coherent sinusoidally oscillating voltage with the amplitude, $V_{\mathrm{ac}}=100 \mathrm{mV}_{\mathrm{p}-\mathrm{p}}$ and various phase, $\phi$. The curves were taken on a Si substrate with natural oxide $\mathrm{SiO}_{2}$. A fitted curve with a parabola for $\Delta f-V_{\text {bias }}$ curves (eq. 12) or a linear line for $g$ - $V_{\text {bias }}$ curves (eq. 13) is overlaid on each experimental curve, indicating a very good agreement between the theory and experiments. As can be seen in Fig. 2(a) and (b), the position of the parabola vertex shifts and the slope of $g$ - $V_{\text {bias }}$ curve changes systematically with varying phase.

In order to further validate the theoretical analysis, the voltage for parabola maximum of $\Delta f-V_{\text {bias }}$ curves and the slope of $g$ - $V_{\text {bias }}$ curves are plotted against the phase, $\phi$, in Fig. 3. Each plot is overlaid with a fitted curve (solid curve) with the cosine function (eq. 12 for the parabola maximum and with the sine function (eq. 13) for the dissipation slope, demonstrating an excellent agreement between the experiment and theory. The voltage for parabola maximum versus phase curve intersects the value for the parabola without ac bias voltage at the phase 

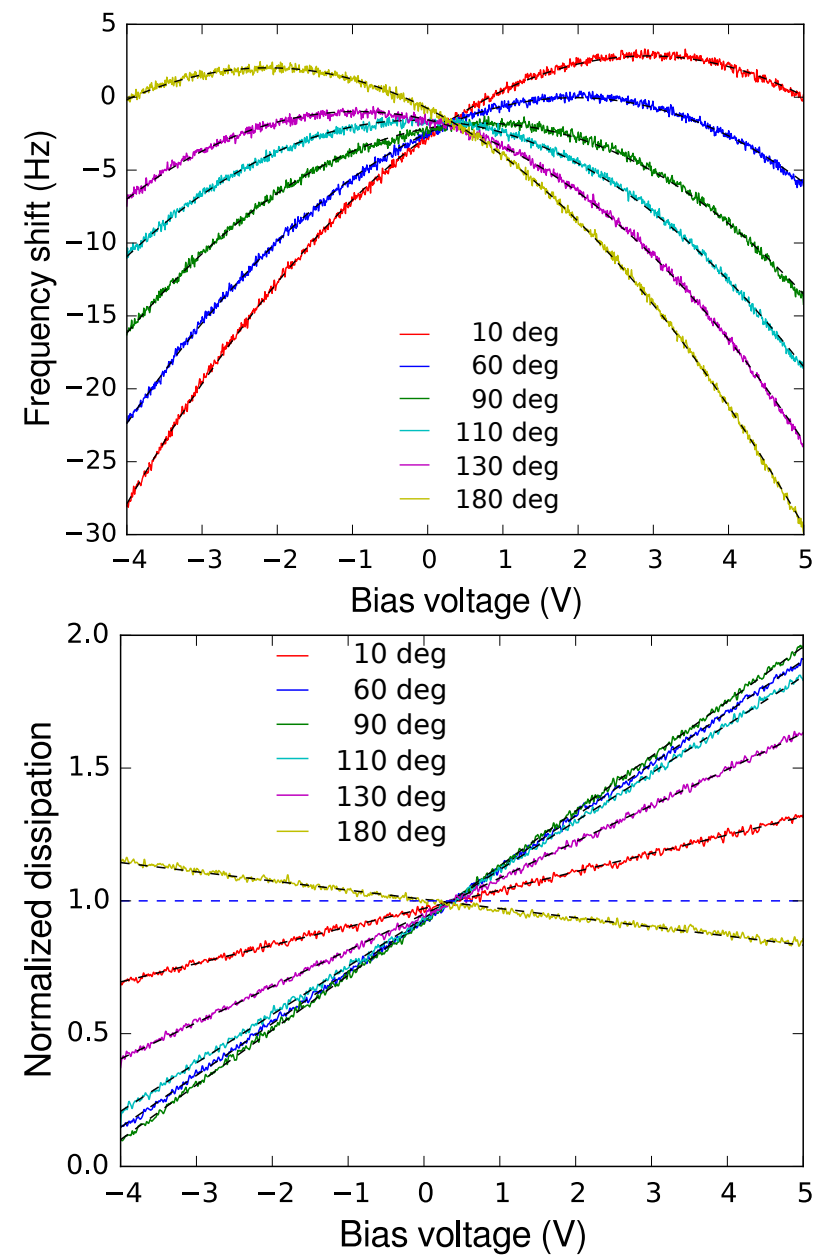

Figure 2. (a) Frequency shift, $\Delta f$, and (b) dissipation signal, $g$, versus dc bias voltage, $V_{\text {bias }}$, curves taken with a coherent sinusoidally oscillating voltage with the amplitude, $V_{\mathrm{ac}}=100 \mathrm{mV}_{\mathrm{p}-\mathrm{p}}$ and various phases, $\phi$, applied to a $200 \mathrm{~nm}$ thick $\mathrm{SiO}_{2}$ on $\mathrm{Si}$ substrate. The dissipation signal is normalized with the value without the ac bias voltage (indicated with the horizontal blue dashed line). In both figures, each of dashed lines represent fitted curves assuming a parabola for $\Delta f$ and a linear line for $g$ as indicated in eq. 12 and 13 respectively. The oscillation amplitude of the tip was $7.2 \mathrm{~nm}_{\mathrm{p}-\mathrm{p}}$ and the quality factor of the cantilever was 9,046 .

of $97^{\circ}$ as opposed to $90^{\circ}$ which is predicted by the theory. This deviation is mainly due to the phase delay in the photodiode preamplifer electronics. The dissipation slope takes its maximum value at around $81^{\circ}$, again deviating from the theoretical value of $90^{\circ}$. This deviation is probably due to the residual capacitive crosstalk to the excitation piezo [14, 15].

Figure 4 shows topography and potential images of a patterned $\mathrm{MoS}_{2}$ on $\mathrm{SiO}_{2} / \mathrm{Si}$ substrate taken by (a) D-KPFM and (b) FM-KPFM techniques with the same tip. In D-KPFM imaging, a sinusoidally oscillating voltage with an amplitude of $V_{\mathrm{ac}}=80 \mathrm{mV}_{\mathrm{p}-\mathrm{p}}$ phase-locked with the tip oscillation was applied to the sample. In FM-KPFM imaging, a sinusoidally oscillating voltage with the amplitude of $V_{\mathrm{ac}}=1.0 \mathrm{Vp}-\mathrm{p}$ and frequency of $300 \mathrm{~Hz}$ was applied to the sample. The scan-

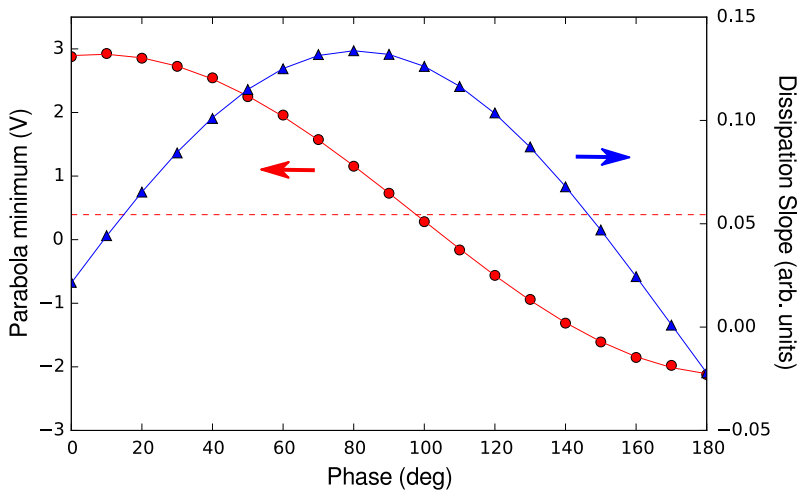

Figure 3. Voltage of minimum of the measured $\Delta f-V_{\text {bias }}$ curves (red circles) (Fig. 2 a) ) and the slope of dissipation- $V_{\text {bias }}$ curves (blue circles) (Fig. 2 b)). Each solid line represents the fitted curve with the cosine function (eq. 12) for the parabola minimum and with the sine function (eq. 13) for the dissipation slope. The horizontal dashed line indicates the voltage for parabola minimum without the ac bias voltage.

ning time for D-KPFM and FM-KPFM imaging were 1 and 2 s/line, respectively. The number of pixels of the images is $512 \times 512$.

A flake of $\mathrm{MoS}_{2}$ was deposited onto a $\mathrm{SiO}_{2} / \mathrm{Si}$ substrate by mechanical exfoliation and a stripe pattern was created by reactive ion etching on top of the flake. The topography images show an unetched ridge located between the etched regions. The height of the ridge is approximately $20 \mathrm{~nm}$ with respect to the etched regions. A clear fractal-like pattern can be seen on the ridge in both potential images. The potential contrast can be ascribed to the residue of the etch resist (PMMA) as the topography images show the similar contrast whose thickness is about $1 \mathrm{~nm}$.

Although both potential images taken with D-KPFM and FM-KPFM show a very similar pattern on the ridge, we notice lower contrast in the potential image taken with D-KPFM than that with FM-KPFM. The difference is more clearly seen in the line profile attached for each potential image. The peakto-peak value of the potential variation in the D-KPFM image is $\sim 0.15 \mathrm{~V}$, about one third that in the FM-KPFM image $(\sim 0.5 \mathrm{~V})$. The similar difference has been observed in the potential contrast taken with FM-KPFM and AM-KPFM and is ascribed to the fact that the AM-KPFM is sensitive to electrostatic force whereas FM-KPFM uses the modulation in the resonance frequency shift which is sensitive to force gradient [4, 17]. The similarity between D-KPFM and AM-KPFM is apparent in the expression of $g$ (eq. 17) which is proportional to $\alpha_{0}=-\left.\frac{1}{2} \frac{\partial C}{\partial z}\right|_{z_{0}}$ rather than $\alpha^{\prime}$. This indicates that the smaller potential variation observed in D-KPFM resulted from larger spatial average due to the stray capacitance including the body of the tip and the cantilever [18, 19].

In spite of lower contrast, D-KPFM has a clear advantage that it requires much smaller $V_{\mathrm{ac}}=80 \mathrm{mV}_{\mathrm{p}-\mathrm{p}}$ compared with $1 \mathrm{~V}_{\mathrm{p}-\mathrm{p}}$ for FM-KPFM, in this case. This advantage is important for such samples as semiconductor where the influence 
(a) D-KPFM

Topography

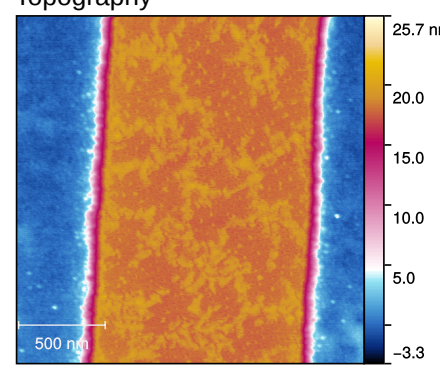

Potential
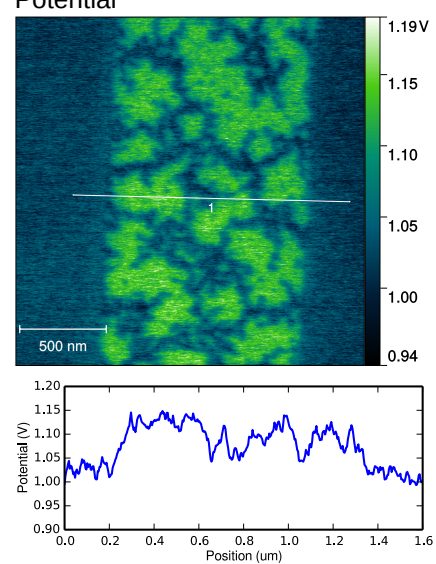

(b) FM-KPFM

Topography

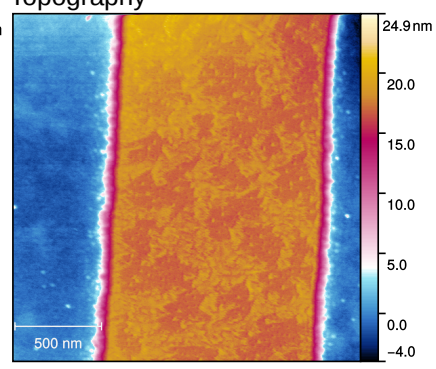

Potential
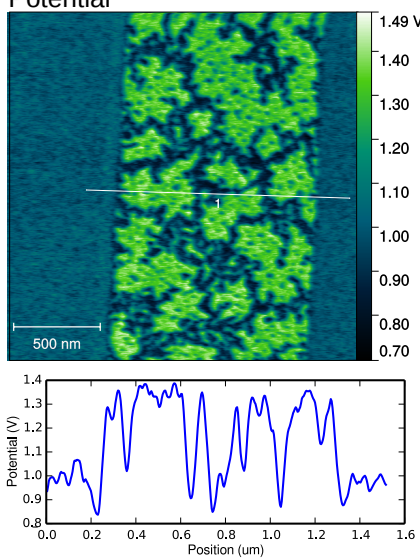

Figure 4. Simultaneously taken topography and potential images of patterned $\mathrm{MoS}_{2}$ on $\mathrm{SiO}_{2} / \mathrm{Si}$ substrate by (a) D-KPFM $(\Delta f=$ $-4.2 \mathrm{~Hz}, A=5 \mathrm{~nm}_{\mathrm{p}-\mathrm{p}}, V_{\mathrm{ac}}=80 \mathrm{mV}_{\mathrm{p}-\mathrm{p}}$ ) and (b) FM-KPFM techniques $\left(\Delta f=-4.2 \mathrm{~Hz}, A=5 \mathrm{~nm}_{\mathrm{p}-\mathrm{p}}, V_{\mathrm{ac}}=1.0 \mathrm{~V}_{\mathrm{p}-\mathrm{p}}, f_{\mathrm{ac}}=300 \mathrm{~Hz}\right)$.

of the large $V_{\mathrm{ac}}$ can be very important due to band-bending effects.

The detection bandwidth of D-KPFM is determined by the bandwidth of the amplitude control feedback loop used in FMKPFM. In fact, applying the coherent $V_{\text {ac }}$ causing dissipative force can be used to measure the dynamics of the amplitude control feedback system. In FM-KPFM, the AFM cantilever serves for the frequency determining element of an oscillator circuit (i.e.self-oscillator) so that the oscillation frequency of the oscillator keeps track of the resonance frequency of the cantilever. In this way, the conservative force has no influence on the drive amplitude [20] and the amplitude controller compensates for the effective $Q$ factor change caused by dissipative force. Therefore, by modulating $V_{\text {bias }}$ at a low frequency (< a few $\mathrm{kHz}$ ) together with applying the coherent $V_{\mathrm{ac}}$, the amplitude of the dissipative force can be modulated as can been seen in eq. 15 and the frequency response of the amplitude feedback loop can thus be measured with a lock-in amplifier. The measured $-3 \mathrm{~dB}$ bandwidth of the amplitude feedback loop is as high as $1 \mathrm{kHz}$, which is wider than that of the PLL frequency detector $(400 \mathrm{~Hz})$. Note that in contrast to the settling time of the oscillation amplitude of a cantilever subject to a change in conservative force which is $\tau \sim Q / f_{0}$ [21], the response time to a dissipative force is not limited by $Q$ and can be faster as the energy dissipated per cycle is given by $\pi F_{\text {quad }} A$ rather than $\pi k A^{2} / Q$ for the case of conservative force. This explains the observed fast response of the amplitude feedback loop, resulting in the wider bandwidth of the voltage feedback loop in D-KPFM than that in FM-KPFM that is limited by PLL demodulation bandwidth (typically $<1 \mathrm{kHz}$ ) which sets the bias modulation frequency.

The noise of $V_{\text {cpd }}$ is ultimately determined by the noise in the tip oscillation amplitude, $\delta A$. The change in the oscillation amplitude of the self-excited cantilever, $\Delta A$, caused by the dissipative force, $F_{\text {quad }}$, is given by

$$
\Delta A=-\frac{Q}{k} F_{\text {quad }}=-\frac{Q}{k} \alpha_{0} V_{\mathrm{dc}} V_{\mathrm{ac}}=\left.\frac{Q}{k} \frac{\partial C}{\partial z}\right|_{z_{0}} V_{\mathrm{dc}} V_{\mathrm{ac}}
$$

Eq. 18 resembles the amplitude response of a simple harmonic oscillator driven on resonance on which AM-KPFM is based.

The noise in $V_{\text {cpd }}, \delta V_{\text {cpd }}$ can thus be expressed as follows:

$$
\delta V_{\mathrm{dc}}=\frac{k}{Q} \frac{\delta A}{\left.\frac{\partial C}{\partial z}\right|_{z_{0}} V_{\mathrm{ac}}}
$$

which agrees with the result by Fukuma et al. [8]. This indicates that $\delta V_{\mathrm{cpd}}$ is proportional to $k / Q$. In typical AM-KPFM measurements [17] where the second flexural mode oscillation is used for detecting electrostatic force, the improvement of $\delta V_{\text {cpd }}$ by the enhanced $Q$ factor is partially cancelled by the substantially higher dynamic spring constant of the second mode with $k_{2 \text { nd }} \approx 40 k$ [22]. D-KPFM enables to fully take advantage of the resonance enhancement while retaining the advantages of the single-pass FM-AFM.

So far we assumed that no other process other than the dissipative electrostatic force causes the dissipation signal in FMAFM systems. Although it is often the case that the contribution from other processes are negligible, even when other intrinsic or extrinsic dissipation processes are present, it is possible to separate the dissipation induced by $F_{\text {quad }}$ just as is done in FM-KPFM with $\Delta f$ or in the method by Fukuma et al. [8]. In this case $V_{\text {bias }}$ needs to be modulated at a low frequency and the resulting modulated dissipation signal is used for the dc bias feedback. Clearly this scheme is slower than the fast response achieved by D-KPFM technique.

In conclusion, we report a new experimental technique for Kelvin probe force microscopy using the dissipation signal of FM-AFM for dc voltage feedback. It features the simpler implementation and faster scanning as it requires no low frequency modulation. The dissipation is caused by the oscillating electrostatic force that is coherent with the tip oscillation, which is induced by a sinusoidally oscillating ac voltage applied between the tip and sample. We analyzed the effect of the phase of the oscillating force on the frequency shift and dissipation and found that the relative phase of $90^{\circ}$ is the most appropriate for KPFM measurements. D-KPFM technique requires a significantly smaller ac voltage amplitude (a few tens of $\mathrm{mV}$ ) by virtue of the resonance enhanced force detection 
and the use of fundamental flexural mode oscillation. This feature will be useful in the electrical characterizations of materials whose electrical properties are sensitive to the externally applied electric field.

The authors would like to thank Dr. Omid Salehzadeh Einabad and Prof. Zetian Mi at McGill University for providing the $\mathrm{MoS}_{2}$ sample. This work was partly supported by the Natural Science and Engineering Research Council (NSERC), le Fonds Québécois de Recherche sur la Nature et les Technologies (FQRNT).

\section{REFERENCES}

* Corresponding author yoichi.miyahara@ mcgill.ca

[1] M. Nonnenmacher, M. P. O'Boyle, and H. K. Wickramasinghe, Appl. Phys. Lett. 58, 2921 (1991)

[2] A. Kikukawa, S. Hosaka, and R. Imura, Rev. Sci. Instrum. 67, 1463 (1996)

[3] C. Sommerhalter, T. W. Matthes, T. Glatzel, A. Jäger-Waldau, and M. C. Lux-Steiner, Appl. Phys. Lett. 75, 286 (1999)

[4] U. Zerweck, C. Loppacher, T. Otto, S. Grafstrom, and L. M. Eng, Phys. Rev. B 71, 125424 (2005).

[5] S. Kitamura, K. Suzuki, and M. Iwatsuki, Appl. Surf. Sci. 140, 265 (1999)

[6] Z. Schumacher, Y. Miyahara, L. Aeschimann, and P. Grütter, Beilstein Journal of Nanotechnology 6, 1450 (2015)
[7] S. A. Burke, J. M. LeDue, Y. Miyahara, J. M. Topple, S. Fostner, and P. Grutter, Nanotechnology 20, 264012 (2009)

[8] T. Fukuma, K. Kobayashi, H. Yamada, and K. Matsushige, Rev. Sci. Instrum. 75, 4589 (2004)

[9] L N Kantorovich et al, J. Phys. Condens. Matter 12, 795 (2000)

[10] H. Hölscher, B. Gotsmann, W. Allers, U. Schwarz, H. Fuchs, and R. Wiesendanger, Phys. Rev. B 64, 075402 (2001)

[11] L. Kantorovich and T. Trevethan, Phys. Rev. Lett. 93, 1 (2004)

[12] J. E. Sader, T. Uchihashi, M. J. Higgins, A. Farrell, Y. Nakayama, and S. P. Jarvis, Nanotechnology 16, S94 (2005)

[13] T. Fukuma, M. Kimura, K. Kobayashi, K. Matsushige, and H. Yamada, Review of Scientific Instruments 76, 53704 (2005)

[14] T. Mélin, S. Barbet, H. Diesinger, D. Théron, and D. Deresmes, Rev. Sci. Instrum. 82, 036101 (2011)

[15] H. Diesinger and D. Deresmes, in Kelvin Probe Force Microsc. Springer Series in Surface Sciences, Vol. 48, edited by S. Sadewasser and T. Glatzel (Springer Berlin Heidelberg, Berlin, Heidelberg, 2012) pp. 25-44.

[16] P. Zahl, T. Wagner, R. Möller, and A. Klust, J. Vac. Sci. Technol. B Microelectron. Nanom. Struct. 28, C4E39 (2010)

[17] T. Glatzel, S. Sadewasser, and M. Lux-Steiner, Appl. Surf. Sci. 210, 84 (2003)

[18] T. Hochwitz, A. K. Henning, C. Levey, and C. Daghlian, Journal of Vacuum Science \& Technology B: Microelectronics and Nanometer Structures 14, 457 (1996)

[19] H. O. Jacobs, P. Leuchtmann, O. J. Homan, and A. Stemmer, J. Appl. Phys. 84, 1168 (1998).

[20] A. Labuda, Y. Miyahara, L. Cockins, and P. Grütter, Physical Review B 84, 125433 (2011).

[21] T. R. Albrecht, P. Grutter, D. Horne, and D. Rugar, J. Appl. Phys. 69, 668 (1991)

[22] J. Melcher, S. Hu, and A. Raman, Appl. Phys. Lett. 91, 53101 (2007) 